\title{
Leveraging GIS Technologies for Informed Decision-making in COVID-19 Pandemic
}

\author{
Rakibul Ahasan, Md. Mahbub Hossain
}

\author{
Author name [1]: Rakibul Ahasan \\ Affiliation: Department of Geography, Texas A\&M University, College Station, Texas, USA. \\ Full Contact Information: Department of Geography, Texas A\&M University, College Station, 3147 \\ TAMU, 810 Eller O\&M Building, College Station, Texas 77843, USA. \\ Phone: 1 (515) 708-3522 \\ Email: rahasan@tamu.edu
}

Author name [2]: Md. Mahbub Hossain

Affiliation: Department of Health Promotion and Community Health Sciences, Texas A\&M University, College Station, Texas, USA.

Full Contact Information: Department of Health Promotion and Community Health Sciences, Texas A\&M School of Public Health, TX 77843, USA.

Phone: 1 (979) 739-7014

Email:mhossain@tamu.edu 
With gradually increasing number of confirmed cases and case-fatalities, the coronavirus disease (COVID-19) pandemic had changed the dynamics of human lives globally (World Health Organization (WHO) 2020). Different Geographic Information System (GIS) techniques are widely used across scientific disciplines, including public health since the mid-1960s. A study published in 2014 reported that one-fourth of the studies out of the reviewed 829 articles used GIS-based applications, especially for infectious disease mapping (Lyseen, Nøhr et al. 2014). Recently, GIS has been playing a critical role in understanding the spatial clustering and transmission trends of the ongoing COVID-19 (Dong, Du et al. 2020). However, it can be argued that the applications of GIS technologies could have provided more insights for research and practice in the context of COVID-19 pandemic.

One of the most reliable documentation and near-real-time GIS-based tracking of COVID-19 cases was created and maintained by the Johns Hopkins University. The online dashboard and near-live tracking of cases and COVID-19 related fatalities was not only the first of its kind but also aided the scientific community and practitioners in comprehending the magnitude of the pandemic during the early days (Dong, Du et al. 2020). Another example of early application of GIS in COVID-19 related studies was to determine whether meteorological factors have any association in the virus transmission. Few studies used different linear regression and Geographically Weighted Regression (GWR) techniques and correlation analysis techniques to examine the relation between COVID-19 cases and temperature, wind speed, solar radiation, daylight hours, and humidity ( $\mathrm{Wu}$, Jing et al. 2020). Although, none of these studies found any confirmed correlation between temperature or humidity and COVID-19 transmission. Further applications of GIS in COVID-19 related studies were focused on either visualizing the case counts on maps or displaying it based on different administrative boundaries. However, there are a few studies that used GIS for hotspot analysis to identify the concentration of confirmed cases or deaths or vulnerable locations. These hotspot analyses not only helped to identify the spatial clustering of the incidences but also aided in identifying the clusters of vulnerable groups, regions needed immediate action, and clusters of cases among fast responders and hospital workers (Paez, Lopez et al. 2020).

Among other spatial analysis techniques, proximity analysis was used by a few studies to identify the distances to the nearest health care facilities. These studies used network analysis to calculate the accessibility to health care facilities and resources to determine the resiliency level of any cities (Jovanović, Klimek et al. 2020). Global and Local Moran's Index, Spatial Autocorrelation Indices, Local Indicators of Spatial Association (LISA) model were similarly used to determine the clusters of confirmed cases or how different communities are affected by the pandemic and whether there was any relation between race and COVID-19 mortality rate. The study in the Chicago area was found that the COVID-19 related mortality rate was much higher among the African American communities, and it has a relation with minority status and language as well (Kim and Bostwick 2020).

Few studies also used satellite images, particulate matter concentration $\left(\mathrm{NO}_{2}, \mathrm{CO}, \mathrm{SO}_{2}, \mathrm{CO}_{2}\right)$, and Air Quality Index (AQI) to compare how air quality had changed during this pandemic. One of the studies in China found that the usual trend of $\mathrm{NO}_{2}$ and $\mathrm{SO}_{2}$ concentrations decrease before the festival and increase afterward was not noticed in 2020 (Fan, Li et al. 2020). Similar studies on human mobility and transportation activities found a reduction in vessel activity in the lockdown period in the European Union maritime regions. Although social distancing and spatial transmission is the most crucial aspect of this pandemic, GIS was not used extensively. At the same time, as we are moving to a world after COVID-19, GIS and other spatial analysis techniques could be of incredible help for the policymakers to adopt different spatially explicit policies during this pandemic as well as in the post-pandemic world.

So far, only one study had used geospatial analysis to identify Spatio-temporal clusters and prediction modeling for COVID-19 transmission. They utilized the Poisson probability distribution model, Kernel density analysis, and space-time scanning analysis to identify high-risk Spatio-temporal clusters for transmission of COVID-19 in Brazil and detected active Spatio-temporal clusters comprising six municipalities in the south-central region of Brazil (Andrade, Gomes et al. 2020). It is well accepted by now that travel restrictions helped to slow down the COVID-19 spread to some extent. However, only 
two studies so far studied how travel restrictions may have limited the epidemic trajectory. One of them developed a global model based on internationally reported cases and mobility data, to project the impact of travel limitations on the national and international spread of the epidemic and revealed that Wuhan travel ban only hindered the overall epidemic trajectory by 3 to 5 days in other cities of China but had a significant influence on the international scale dispersion (Chinazzi, Davis et al. 2020). As the global nations and respective economies are thriving to resume their regular socioeconomic activities resulting in internal population mobility and international travels, it would be useful to adopt spatial-modeling to predict the best and worst-case scenarios and prepare accordingly.

As the world is passing through initial waves of COVID-19 and experts are predicting subsequent waves of cases in different contexts, it is essential that countries prepare themselves with better public health surveillance and contract-tracing techniques. The integration of GIS-based surveillance methods will provide greater flexibility and efficiency to prevent any outbreak and track the cases in a near-realtime manner. China has already employed a social-media based contract tracing app in March. Integration of GIS in such applications will help not only identify the cases or clusters but also trace other people in contact with the patients and keep them in quarantine. The ArcGIS and other open-source GIS software are providing web-based application development options, which could be of great advantage for more accurate and geocoded surveillance and contact tracing.

One of the effective uses of GIS is to predict spatially explicit growth, and which necessitates further attention of the academic and professional communities working on COVID-19. GIS has not been used widely to track the transmission pattern and to predict the transmission at the initial stages. It is essential to leverage GIS to predict not only the confirmed case numbers but also specific locations where the outbreak would happen with higher statistical precision. Although both suitability analysis and hotspot analysis were used to some extent, those could be further used to identify health care facilities, as well as quarantine sites, informing local and regional resource mapping and subsequent planning. Similarly, satellite images and processed rasters could be used in GIS to identify the changes in Green House Gas (GHG) compositions, Urban Heat Island (UHI) effects and energy consumption patterns, and epidemiological clusters. Such GIS-based modeling of the spatially-explicit numbers and growth direction may enable the policymakers to adapt context and location-specific policy measure to prevent outbreaks, isolate infections, minimize community transmission, enforce public health guidelines, whenever necessary.

We declare no competing interests.

\section{References}

Andrade, L. A., D. S. Gomes, M. A. d. O. Góes, M. S. F. d. Souza, D. C. P. Teixeira, C. J. N. Ribeiro, J. A. B. Alves, K. C. G. M. d. Araújo and A. D. d. Santos (2020). "Surveillance of the first cases of COVID-19 in Sergipe using a prospective spatiotemporal analysis: the spatial dispersion and its public health implications." Revista da Sociedade Brasileira de Medicina Tropical 53.

Chinazzi, M., J. T. Davis, M. Ajelli, C. Gioannini, M. Litvinova, S. Merler, A. P. y Piontti, K. Mu, L. Rossi and K. Sun (2020). "The effect of travel restrictions on the spread of the 2019 novel coronavirus (COVID19) outbreak." Science 368(6489): 395-400.

Dong, E., H. Du and L. Gardner (2020). "An interactive web-based dashboard to track COVID-19 in real time." The Lancet Infectious Diseases 20(5): 533-534.

Fan, C., Y. Li, J. Guang, Z. Li, A. Elnashar, M. Allam and G. de Leeuw (2020). "The Impact of the Control Measures during the COVID-19 Outbreak on Air Pollution in China." Remote Sensing 12(10): 1613. 
Jovanović, A., P. Klimek, O. Renn, R. Schneider, K. Øien, J. Brown, M. DiGennaro, Y. Liu, V. Pfau and M. Jelić (2020). "Assessing resilience of healthcare infrastructure exposed to COVID-19: emerging risks, resilience indicators, interdependencies and international standards." Environment Systems \& Decisions: 1.

Kim, S. J. and W. Bostwick (2020). "Social Vulnerability and Racial Inequality in COVID-19 Deaths in Chicago." Health Education \& Behavior: the Official Publication of the Society for Public Health Education: 1090198120929677-1090198120929677.

Lyseen, A. K., C. Nøhr, E. M. Sørensen, O. Gudes, E. M. Geraghty, N. T. Shaw, C. Bivona-Tellez and I. H. G. W. Group (2014). "A Review and Framework for Categorizing Current Research and Development in Health Related Geographical Information Systems (GIS) Studies." Yearbook of medical informatics 9(1): 110-124.

Paez, A., F. A. Lopez, T. Menezes, R. Cavalcanti and M. G. d. R. Pitta (2020). "A Spatio-Temporal Analysis of the Environmental Correlates of COVID-19 Incidence in Spain." Geographical Analysis.

World Health Organization (WHO) (2020). "Coronavirus disease 2019 ( COVID-19): situation report, 201."

Wu, Y., W. Jing, J. Liu, Q. Ma, J. Yuan, Y. Wang, M. Du and M. Liu (2020). "Effects of temperature and humidity on the daily new cases and new deaths of COVID-19 in 166 countries." Science of the Total Environment: 139051. 